\title{
Probing Local Environments by Time-Resolved Stimulated Emission Spectroscopy
}

\author{
Ana Rei, ${ }^{1}$ Graham Hungerford, ${ }^{2}$ Michael Belsley, ${ }^{1}$ M. Isabel C. Ferreira, ${ }^{1}$ \\ and Peter Schellenberg ${ }^{1}$ \\ ${ }^{1}$ Centro de Física, Universidade do Minho, Campus de Gualtar, 4710-057 Braga, Portugal \\ ${ }^{2}$ HORIBA Jobin Yvon IBH Ltd., Skypark 5, 45 Finnieston Street, Glasgow G3 8JU, UK
}

Correspondence should be addressed to Peter Schellenberg, peter.schellenberg@fisica.uminho.pt

Received 31 July 2011; Revised 22 September 2011; Accepted 22 September 2011

Academic Editor: A. M. Brouwer

Copyright ( 2012 Ana Rei et al. This is an open access article distributed under the Creative Commons Attribution License, which permits unrestricted use, distribution, and reproduction in any medium, provided the original work is properly cited.

Time-resolved stimulated emission spectroscopy was employed to probe the local environment of DASPMI (4-(4-(dimethylamino)styryl)-N-methyl-pyridinium iodide) in binary solvents of different viscosity and in a sol-gel matrix. DASPMI is one of the molecules of choice to probe local environments, and the dependence of its fluorescence emission decay on viscosity has been previously used for this purpose in biological samples, solid matrices as well as in solution. The results presented in this paper show that time-resolved stimulated emission of DASPMI is a suitable means to probe the viscosity of local environments. Having the advantage of a higher time resolution, stimulated emission can provide information that is complementary to that obtained from fluorescence decay measurements, making it feasible to probe systems with lower viscosity.

\section{Introduction}

Fluorescent stilbenoid dyes are among the classes of chromophores most widely used in staining biological samples to be investigated by fluorescence microscopy methods [1-4]. Stilbenoid dyes show complex excited state dynamics, such as isomerisation processes, which are often accompanied by the emergence of charge transfer states [2, 5-9]. An important consequence of these dynamics is that their fluorescence properties are particularly sensitive to the local environment, which not only increases the contrast between sample features, but also delivers specific information on local properties. In the particular case of DASPMI (4(4-(dimethylamino)styryl)-N-methyl-pyridinium iodide), solvent polarity as well as viscosity can influence the fluorescence properties of this dye. To a good approximation, the peak of the emission spectrum can be used as an indicator for the polarity of its surroundings, while the fluorescence lifetime varies with the viscosity of the local environment $[8,9]$.

It has been previously demonstrated that the fast molecular dynamics processes in DASPMI, such as the rearrangements of the dye upon transitions to intramolecular charge transfer states, are sensitive to the local viscosity $[2,10$, 11]. In particular, the fluorescence lifetime of DASPMI is quite sensitive to the local viscosity, making fluorescence lifetime measurements a method of choice to probe the microheterogeneous environments in confining media such as sol-gel based or biological systems [10, 12, 13]. Previous studies have shown that when DASPMI is incorporated in sol-gel-derived media, the lifetime of the fastest decay component can increase up to a hundred fold while the lifetime of the longer decay components tend to stabilize [13].

Sol-gel-derived media are promising materials for applications as optical biosensors [14-16]. Their porosity, robustness, transparency in the near UV and visible part of the spectrum along with the inherent flexibility of the sol-gel method have allowed the encapsulation of a wide range of biomolecules, including proteins, DNA, and even whole cells $[17,18]$, and their structure allows for a limited substance mobility within the amorphous porous network. Other directions of research are motivated by the requirement to produce new materials to be used in electronics, communication, energy, and other high-technology fields $[19,20]$. The encapsulation of enzymes is particularly attractive because of 
the efficiency, specificity, and selectivity of these biocatalysts. There are many publications on the successful entrapment of enzymes and for a review see [21]; however, some major issues still remain, explaining why this is still an intense field of investigation. Knowledge about the microenvironment composition inside the pores and about the molecular mobility of the enzyme and substrates/products of reaction is crucial to comprehend the enzyme kinetics in confining media. In this frame, measurements concerning the viscosity evolution in a sol-gel sample would be important for characterizing mass transport inside the matrix [14, 22].

In the present work, we report on complementary measurements employing femtosecond time-resolved stimulated emission to elucidate the DASPMI kinetics in homogeneous solution and a microheterogeneous sol-gel medium. As the experimental time resolution in these techniques is of order $100 \mathrm{fs}$, the technique could be employed to probe faster decay processes compared to fluorescence lifetime measurements. This would allow one to explore the faster kinetics affiliated with lower viscosity matrices, extending the range of the technique.

In this work, the principal applicability of the method is tested. Apart from measurements on matrices produced by the sol-gel path, we probe glycerol-water mixtures of various viscosities. As was the case for the fluorescence decay signals using fluorescence upconversion experiments [23, 24 ], we are able to identify wavelength-dependent kinetics. With the increased time resolution of an ultrafast laser system, we can clearly identify dynamic processes in DASPMI on a subpicosecond scale.

\section{Experimental Setup}

The pump-probe measurements were carried out using the output of a Coherent Legend Ti:sapphire-chirped pulse regenerative amplifier system seeded by a Coherent Mira Ti: sapphire oscillator which in turn was pumped by a $5 \mathrm{~W}$ frequency-doubled Nd-laser. The system generated a $1 \mathrm{kHz}$ pulse train at a wavelength of $800 \mathrm{~nm}$ with a FWHM of around $120 \mathrm{fs}$ and pulse energies of $2 \mathrm{~mJ}$. A small portion of these amplified pulses were used to generate the excitation pulses centred at $400 \mathrm{~nm}$ by frequency doubling in a BBO crystal. The majority of the pulse intensity is used to generate the probe beam by pumping an ultrafast optical parametric amplifier (OPA) from Light Conversion (TOPAS). The time delay $\Delta t$ between the probe and the excitation beam was scanned with a delay line (Standa, Lithuania 8MT167-100) with minimum step sizes corresponding to a delay of around 4 fs. The signals were collected with a fast PIN-biased photodiode (Thorlabs DET10A/M). A modular boxcar integrator system SR-240/SR-245/SR-250/SR-280 (Stanford Research Systems) was used for sampling and digitizing the signals. For controlling the experiment; a homemade labview program was employed. The data were fitted with the Fluofit program (Picoquant Berlin) employing an artificially created gaussian profile deconvolution function to take into account the finite temporal width of the excitation pulses. Its width was set to $120 \mathrm{fs}$ FWHM, which was derived from the experimental autocorrelation function of the Ti:Sa amplifier output.
The pump and probe beams were made to intersect at an angle of approximately 15 degrees, and the liquid solutions were placed in a $1 \mathrm{~mm}$ glass cuvette centred on the crossing of the pump and probe beams.

The DASPMI dye was purchased from Molecular Probes (Invitrogen, SA), the Glycerol was from Riedel-de Haën, and both were used without further purification.

The sol-gel media were produced as previously described [25], based on the method presented by Flora and Brennan [26], in the form of a monolith. Briefly, the sol was made by mixing $9 \mathrm{~mL}$ of tetraethyl orthosilicate (TEOS) (Aldrich) with $3 \mathrm{~mL}$ of acidic water. This mixture was sonicated for 1 hour and stored at $-18^{\circ} \mathrm{C}$ for about a month. The matrices were produced by taking $2 \mathrm{~mL}$ of the sol and mixing it with $2 \mathrm{~mL}$ of phosphate buffer solution $(\mathrm{pH} 7)$ containing the fluorophore DASPMI. The samples, prepared between two glass plates, were about 1-2 mm thick. The outer edges of the glass plates were sealed with epoxy to prevent solvent evaporation from the freshly prepared gel. The doped sol-gel matrices were used within hours of preparation. Because of photobleaching of the dye, a new probing position was used for each time delay point. Since the samples contained small spatial inhomogeneities, this caused a noticeable increase in the noise level of the sol-gel measurements as compared with those carried out in solution.

\section{Results and Discussion}

The dynamics of DASPMI kinetics were probed by stimulated emission spectroscopy in various glycerol: water mixtures, in pure water, and in matrices produced via the solgel process for probe wavelengths of $560 \mathrm{~nm}$ to $620 \mathrm{~nm}$. The recovered parameters are shown in Table 1 and a majority of the decays are multiexponential in nature.

To exemplify, an illustration of a selected set of decays is given in Figure 1. It shows the time-resolved stimulated emission kinetics of DASPMI in different media at a probe wavelength of $560 \mathrm{~nm}$. The dramatic shortening of the emission decay time upon lowering the viscosity is immediately obvious.

The strong dependence of the stimulated emission decay on the probe wavelength can be seen in Figure 2 for the lowest viscosity solvent (water). In addition to a shortening of the decay time by about a factor of 100 , we also observe a rise time component for probe wavelengths of $600 \mathrm{~nm}$ and $620 \mathrm{~nm}$. Tentatively this may also be present at $580 \mathrm{~nm}$, although it was not possible to obtain a satisfactory fit for a temporally increasing component of the data available at this wavelength. This rise time component may be attributed to a fast excited state relaxation of excited Frank-Condon states, for example, due to a twisted geometry [11], and/or due to solvent relaxation in the excited state [27] particularly as a response to its CT-character. This view is supported by the dramatic increase of the rise time when going from lowviscosity water solution to higher-viscosity glycerol mixtures (Table 1). Interestingly, we could not observe such a rise time in the sol-gel matrixes, which may be attributed to the absence of this motional degree of freedom, although it is 


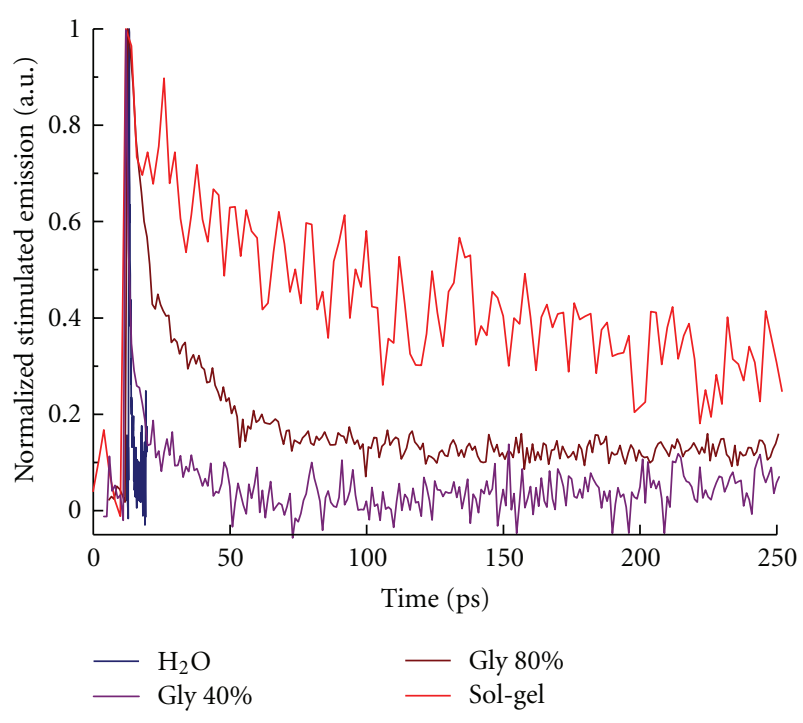

Figure 1: Stimulated emission decay curves of DASPMI in different matrices at a probe wavelength of $560 \mathrm{~nm}$.

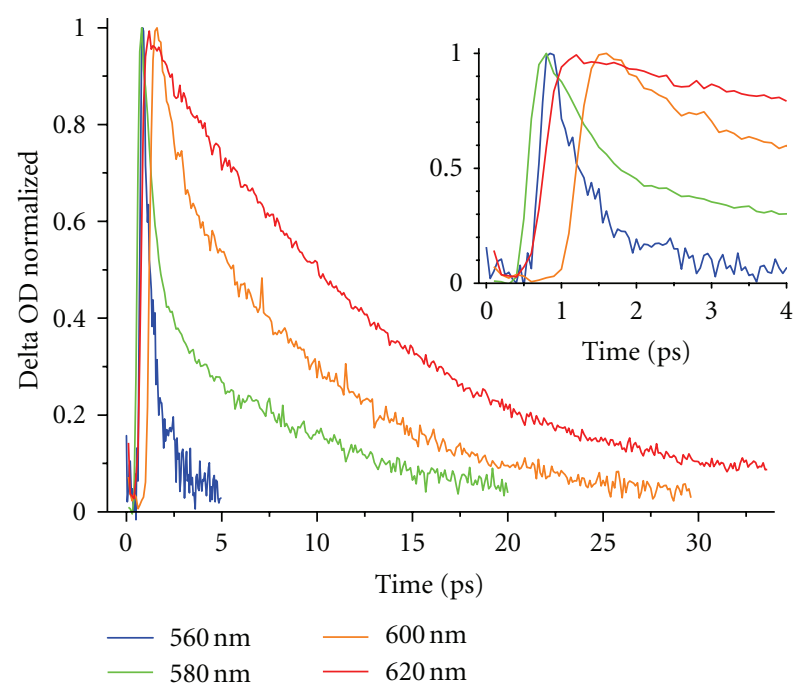

FIGURe 2: Stimulated emission decay curves of DASPMI in water at different probe wavelengths.

also possible that this component evades observation due to the lower signal to noise ratio.

Table 1 summarises all experimentally determined stimulated emission decay fit values for water, two different water: glycerol mixtures (40\% and $80 \%$ glycerol, expressed in mass percent) and the sol-gel system. Empty fields indicate the absence of a decay constant in that time range. The time ranges are arbitrarily chosen and do not necessarily represent the presence or absence of a particular molecular process. It should be borne in mind that, in highly viscous media, inhomogeneous broadening of the kinetic components can occur, leading to dispersive kinetics. Such processes are not explicitly considered here but may contribute to the complex kinetics observed. The timescale of the parameters presented in this table also shows that the use of an ultrafast system allows an increase in the time resolution for emission studies, as well as the possibility for exploring other excited state kinetic processes.

The values shown in Table 1 emphasise that studies with higher time resolution compared to fluorescence decay are more sensitive to probe local viscosity alterations in lowviscosity environments. It should also be noted that, at a viscosity of $45.9 \mathrm{cp}$ [28] represented by a $80 \%$ Glycerol sample, the data approximately match those obtained with previous fluorescence decay measurements [10]. We note, however, that the fastest decay component is shorter in the stimulated emission experiments.

Following the assessment of Strehmel et al. [11], based on fluorescence decay data, the dynamics of DASPMI can be attributed to several processes: the direct transition from the first excited state to the ground state, a relaxation probably due to a planar charge transfer state, and a slow relaxation related to a twisted charge transfer state.

It can be seen from these experiments that in solution the decay time of the shortest lived component, which is attributed to the planar state of DASPMI, increases significantly upon augmenting the viscosity up to $3.1 \mathrm{cp}$. Further increases in viscosity by more than an order of magnitude only change the decay for a factor of two. In contrast, a similar proportional increase in the decay time in the longer lived (here $\tau_{3}$ ) component attributed to a planar CT state is seen at higher viscosities (on passing from 3.18 to $45.9 \mathrm{cP}$ ). In the time-resolved stimulated emission of DASPMI doped into sol-gel-derived media, only a single component is observed. However, in these studies, we could not follow the ultrafast decay kinetics with high precision but instead were limited to the intermediate time regime. This was because, in order to avoid photobleaching of the sample, different probe regions were selected. This led to increased point-to-point fluctuations due to macroscopic sample inhomogeneities. Furthermore, the number of laser pulses that could be used to probe the solid medium was limited by photobleaching, which, in turn, reduced the measurement statistics.

In previous time-resolved fluorescence measurements, the decay kinetics has been reported to be multiexponential, with three different lifetime components [10, 29]. In comparison with those studies, it is possible that the lifetime component found here corresponds to the fast kinetic component in those experiments, but the dynamics of DASPMI in the confined environment may also be due to processes not observed in the homogeneous liquid phase. An indication for this is that the time constant for the direct transition from $S_{1}$ to $S_{0}$ is only weakly dependent on the viscosity, as witnessed by the small increase observed when going from $3.1 \mathrm{cp}$ to $46 \mathrm{cp}$. In the sol-gel media, however, the time constant is between 84 ps and 186 ps, much greater than the less than 20 ps observed in a solution with a viscosity of $46 \mathrm{cp}$. In fact, a strong increase of the lifetime of DASPMI upon inclusion into sol-gel matrices has been observed before [13], and our data give strong evidence that DASPMI is not localized in microcavities of low viscosity solvent. This is particularly noticeable, as the hydrogels used in this study were still in their initial stages of aging, at which the pores network is still forming. 
TABLE 1: Stimulated emission decay times and relative amplitudes for various different water: glycerol mixtures and for the sol-gel matrix at the different probe wavelengths. A negative amplitude indicates a rising rather than a decaying component. The sol-gel data could be fitted to a single exponential function with a residual amount around $25-30 \%$ that did not decay in the time window of the experiment. The viscosity values are taken from [28].

\begin{tabular}{|c|c|c|c|c|c|c|c|}
\hline Sample & Probe $\lambda(\mathrm{nm})$ & $\tau 1(\mathrm{ps})$ & $\alpha 1$ & $\tau 2(\mathrm{ps})$ & $\alpha 2$ & $\tau 3(\mathrm{ps})$ & $\alpha 3$ \\
\hline \multirow{4}{*}{ Water $(0.89 \mathrm{cp})$} & 560 & & & 0.53 & 1 & & \\
\hline & 580 & & & 0.67 & 0.59 & 9.86 & 0.41 \\
\hline & 600 & 0.25 & -1 & 0.57 & 0.54 & 8.46 & 0.46 \\
\hline & 620 & 0.2 & -1 & & & 12.1 & 1 \\
\hline \multirow{4}{*}{ Glycerol 40\% (3.18 cp) } & 560 & & & 7.91 & 1 & & \\
\hline & 580 & & & 1.7 & 0.59 & 34.3 & 0.41 \\
\hline & 600 & 6.0 & -1 & 6.1 & 0.41 & 69.9 & 0.59 \\
\hline & 620 & 5.1 & -1 & 8.3 & 0.60 & 38.5 & 0.40 \\
\hline \multirow{4}{*}{ Glycerol 80\% (45.9 cp) } & 560 & & & 14.2 & 1 & & \\
\hline & 580 & & & 18.9 & 0.12 & 255.7 & 0.88 \\
\hline & 600 & & & & & 203.2 & 1 \\
\hline & 620 & 4.73 & -1 & & & 254 & 1 \\
\hline \multirow{4}{*}{ Sol-gel } & 560 & & & & & 84 & 0.73 \\
\hline & 580 & & & & & 91 & 0.74 \\
\hline & 600 & & & & & 128 & 0.75 \\
\hline & 620 & & & & & 186 & 0.70 \\
\hline
\end{tabular}

Also, note that the longer lifetimes are not seen in these stimulated emission experiments, as the time range was limited due to the length of the optical delay available for these studies. However, the presence of longer excitation dynamics components can be deduced from a residual amplitude of around 25 to $30 \%$ representing excited states which have not decayed within the time window of the experiment.

In the solutions of different viscosity, the deviation in the shorter lifetimes, as compared to those measured by previous fluorescence lifetime measurements [10] could be due to a lower time resolution in time correlated single photoncounting experiments. In the previous measurements, the observed lifetime is derived after deconvolution of a much longer instrument response function. Alternatively the transitions observed in stimulated emission may be different ones compared to those in fluorescence decay. While fluorescence is dominated by the decay of the relaxed excited state, stimulated emission processes can probe the molecule as it evolves from the initial excitation to the relaxed state, thereby providing complementary information on the probe environment.

\section{Conclusion}

Time-dependent stimulated emission was employed to demonstrate the potential of using DASPMI in matrices to characterise local viscosities, both in solution and solgel-derived media. It can be shown that time-resolved stimulated emission experiments are promising for probing local environments in microheterogeneous environments, like polymers and other amorphous systems and biological samples. The sensitivity of the effect can be compared to that of the corresponding method of employing fluorescence lifetime spectroscopy techniques. However, the time scale for stimulated emission experiments is much faster, as it is basically limited by the pulse width of the laser employed, which in our case was around $100 \mathrm{fs}$. Consequently much faster processes can be accurately probed. In DASPMI, the time evolution of the excited state scales with the viscosity of the solvent and an increased temporal resolution allows one to greatly increase the range over which the local viscosity can be probed. Therefore, time-resolved stimulated emission spectroscopy could be a suitable complementary method to time-resolved fluorescence techniques by which to investigate lower viscosity samples.

It is known from previous work on sol-gel systems that there are kinetic processes on longer time scales as accessible in the present stimulated emission setup. In future experiments, it is crucial to identify the physical processes associated with the time constants observed in sol-gel matrices. It is not unreasonable to associate the subnanosecond decay in sol-gel material with the faster reconfiguration processes in lower viscosity solvents as observed in the stimulated emission experiments presented here and in previous studies using fluorescence upconversion $[23,27]$. This would provide additional insight into the interaction of DASPMI in the sol-gel-derived microheterogeneous environment.

\section{Acknowledgments}

The authors acknowledge financial support from Fundação para a Ciência e a Tecnologia (FCT) for the reequipment Grant REEQ/25/FIS/2005. A. Rei was supported from FCT through the Ph.D. Grant SFRH/BD/27933/2006.

\section{References}

[1] R. Ramadass and J. Bereiter-Hahn, "Picosecond fluorescence decay microscopy of DASPMI stained mitochondria in living cells," European Journal Of Cell Biology, vol. 84, pp. 107-108, 2005. 
[2] R. Ramadass and J. Bereiter-Hahn, "Photophysical properties of DASPMI as revealed by spectrally resolved fluorescence decays," Journal of Physical Chemistry B, vol. 111, no. 26, pp. 7681-7690, 2007.

[3] R. Ramadass and J. Bereiter-Hahn, "How DASPMI reveals mitochondrial membrane potential: fluorescence decay kinetics and steady-state anisotropy in living cells," Biophysical Journal, vol. 95, no. 8, pp. 4068-4076, 2008.

[4] R. Ramadass and J. Bereiter-Hahn, "Excited state photophysical properties of DASPMI in solvents and living cells," Biophysical Journal, p. 326A, 2007.

[5] D. Bingemann and N. P. Ernsting, "Femtosecond solvation dynamics determining the band shape of stimulated emission from a polar styryl dye," The Journal of Chemical Physics, vol. 102, no. 7, pp. 2691-2700, 1995.

[6] M. Dekhtyar and W. Rettig, "Charge-transfer transitions in twisted stilbenoids: interchangeable features and generic distinctions of single- and double-bond twists," Journal of Physical Chemistry A, vol. 111, no. 11, pp. 2035-2039, 2007.

[7] W. Rettig, B. Strehmel, and W. Majenz, "The excited states of stilbene and stilbenoid donor-acceptor dye systems. A theoretical study," Chemical Physics, vol. 173, no. 3, pp. 525537, 1993.

[8] M. Sczepan, W. Rettig, A. I. Tolmachev, and V. V. Kurdyukov, "The role of internal twisting in the photophysics of stilbazolium dyes," Physical Chemistry Chemical Physics, vol. 3, no. 17, pp. 3555-3561, 2001.

[9] M. J. van der Meer, H. Zhang, W. Rettig, and M. Glasbeek, "Femto- and picosecond fluorescence studies of solvation and non-radiative deactivation of ionic styryl dyes in liquid solution," Chemical Physics Letters, vol. 320, no. 5-6, pp. 673680, 2000.

[10] A. Rei, G. Hungerford, and M. I. C. Ferreira, "Probing local effects in silica sol-gel media by fluorescence spectroscopy of p-DASPMI," Journal of Physical Chemistry B, vol. 112, no. 29, pp. 8832-8839, 2008.

[11] B. Strehmel, H. Seifeit, and W. Rettig, "Photophysical properties of fluorescence probes. 2. A model of multiple fluorescence for stilbazolium dyes studied by global analysis and quantum chemical calculation," Journal of Physical Chemistry B, vol. 101, no. 12, pp. 2232-2243, 1997.

[12] D. Jóźwik, E. Miller, B. Wandelt, and S. Wysocki, "The styrylpyridine dye for the silane sol-gel transition studies by time-dependent fluorescence," Spectrochimica Acta A, vol. 64, no. 5, pp. 1125-1132, 2006.

[13] G. Hungerford, M. R. Pereira, J. A. Ferreira et al., "Probing $\mathrm{Si}$ and Ti based sol-gel matrices by fluorescence techniques," Journal of Fluorescence, vol. 12, no. 3-4, pp. 397-417, 2002.

[14] G. Hungerford, A. Rei, M. I. C. Ferreira, K. Suhling, and C. Tregidgo, "Diffusion in a sol-gel-derived medium with a view toward biosensor applications," Journal of Physical Chemistry $B$, vol. 111, no. 13, pp. 3558-3562, 2007.

[15] D. Avnir, O. Lev, and J. Livage, "Recent bio-applications of solgel materials," Journal of Materials Chemistry, vol. 16, no. 11, pp. 1013-1030, 2006.

[16] S. Hudson, J. Cooney, and E. Magner, "Proteins in mesoporous silicates," Angewandte Chemie, vol. 47, no. 45, pp. 8582-8594, 2008.

[17] T. Besanger, Y. Zhang, and J. D. Brennan, "Characterization of fluorescent phospholipid liposomes entrapped in sol-gel derived silica," Journal of Physical Chemistry B, vol. 106, no. 41, pp. 10535-10542, 2002.
[18] W. Jin and J. D. Brennan, "Properties and applications of proteins encapsulated within sol-gel derived materials," Analytica Chimica Acta, vol. 461, no. 1, pp. 1-36, 2002.

[19] H. Dislich, "Sol-Gel $1984 \rightarrow 2004$ (?)," Journal of NonCrystalline Solids, vol. 73, no. 1-3, pp. 599-612, 1985.

[20] S. Sakka, "Sol-gel synthesis of glasses: present and future," American Ceramic Society Bulletin, vol. 64, no. 11, pp. 14631466, 1985.

[21] A. C. Pierre, “The sol-gel encapsulation of enzymes," Biocatalysis and Biotransformation, vol. 22, no. 3, pp. 145-170, 2004.

[22] G. Hungerford, A. Rei, M. I. C. Ferreira, C. Tregidgo, and K. Suhling, "Molecular diffusion within sol-gel derived matrices viewed via fluorescence recovery after photobleaching," Photochemical \& Photobiological Sciences, vol. 6, no. 8, pp. 825-828, 2007.

[23] A. M. Jonkman, P. van der Meulen, H. Zhang, and M. Glasbeek, "Subpicosecond solvation relaxation of DASPI in polar liquids," Chemical Physics Letters, vol. 256, no. 1-2, pp. 21-26, 1996.

[24] H. Wang, H. Zhang, W. Rettig, A. I. Tolmachev, and M. Glasbeek, "Femtosecond dynamics of the $S_{2}$ and $S_{1}$ fluorescence of ionic styryl dyes in polar solvents," Physical Chemistry Chemical Physics, vol. 6, no. 13, pp. 3437-3446, 2004.

[25] G. Hungerford, A. Rei, and M. I. C. Ferreira, "Use of fluorescence to monitor the incorporation of horseradish peroxidase into a sol-gel derived medium," Biophysical Chemistry, vol. 120, no. 2, pp. 81-86, 2006.

[26] K. K. Flora and J. D. Brennan, "Characterization of the microenvironments of PRODAN entrapped in tetraethyl orthosilicate derived glasses," Journal of Physical Chemistry B, vol. 105, no. 48, pp. 12003-12010, 2001.

[27] P. van der Meulen, H. Zhang, A. M. Jonkman, and M. Glasbeek, "Subpicosecond solvation relaxation of 4-(dicyanomethylene)-2-methyl-6-(p-(dimethylamino)styryl)-4H-pyran in polar liquids," Journal of Physical Chemistry, vol. 100, no. 13, pp. 5367-5373, 1996.

[28] R. C. Weast, in Handbook Of Chemistry And Physics, CRC, Boca Raton, Fla, USA, 57th edition, 2000.

[29] G. Hungerford, A. Allison, D. McLoskey, M. K. Kuimova, G. Yahioglu, and K. Suhling, "Monitoring Sol-to-Gel transitions via fluorescence lifetime determination using viscosity sensitive fluorescent probes," Journal of Physical Chemistry B, vol. 113, no. 35, pp. 12067-12074, 2009. 


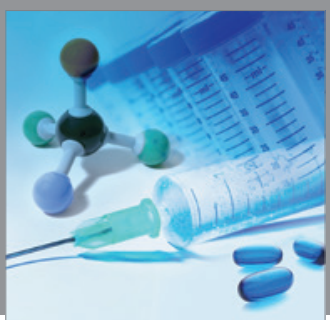

International Journal of

Medicinal Chemistry

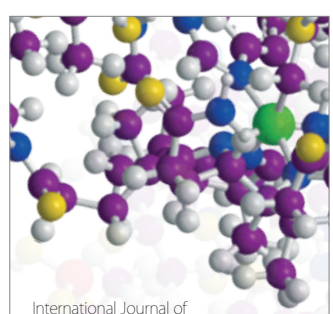

Carbohydrate Chemistry

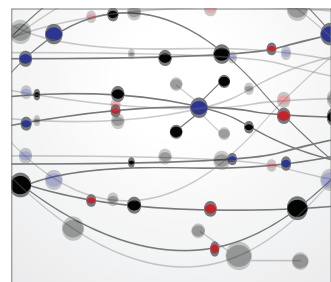

The Scientific World Journal
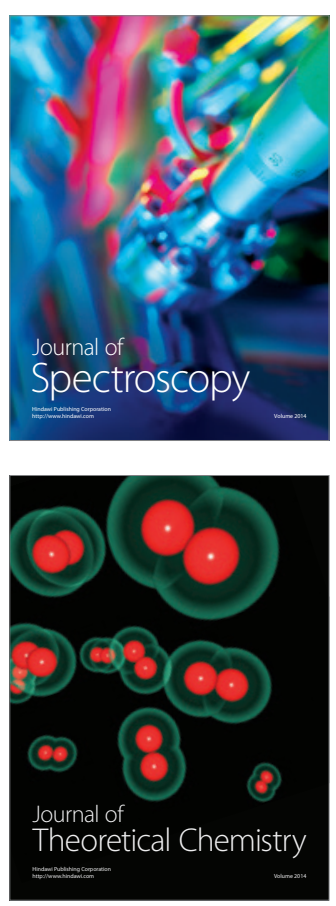
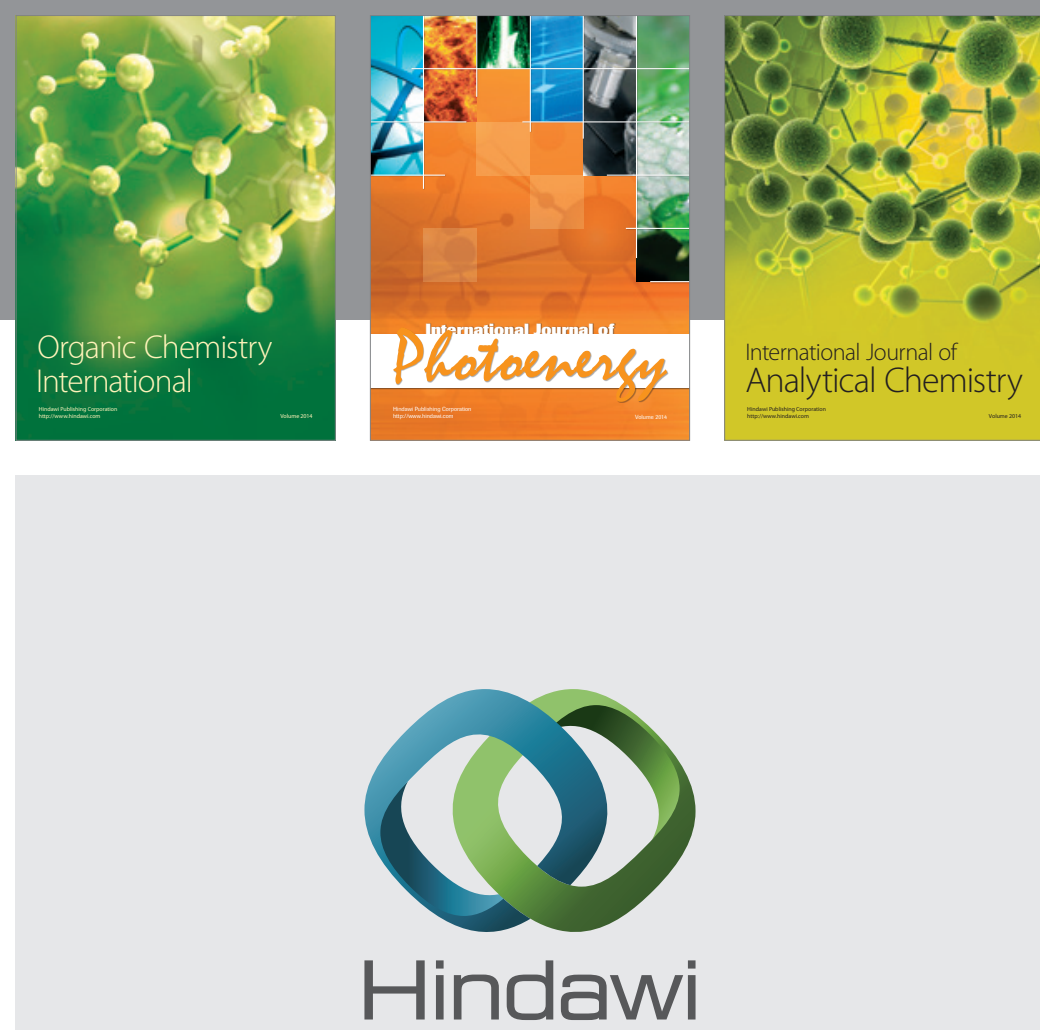

Submit your manuscripts at

http://www.hindawi.com
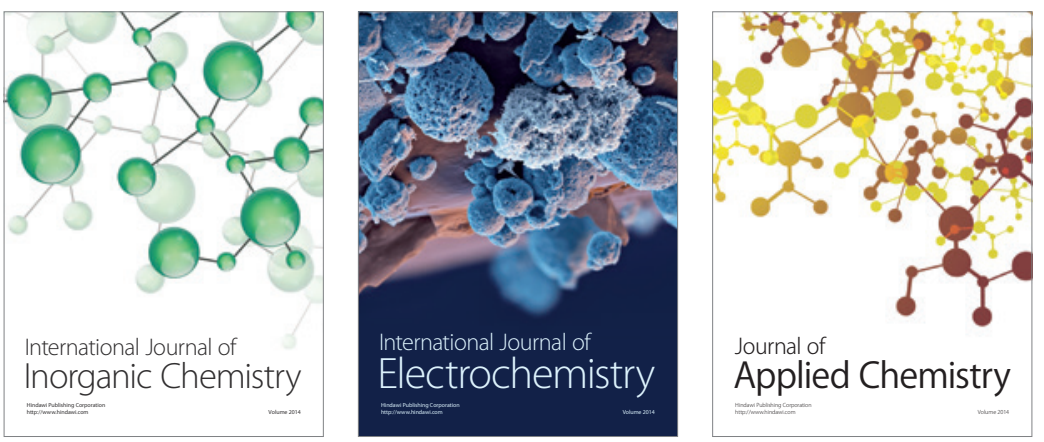

Journal of

Applied Chemistry
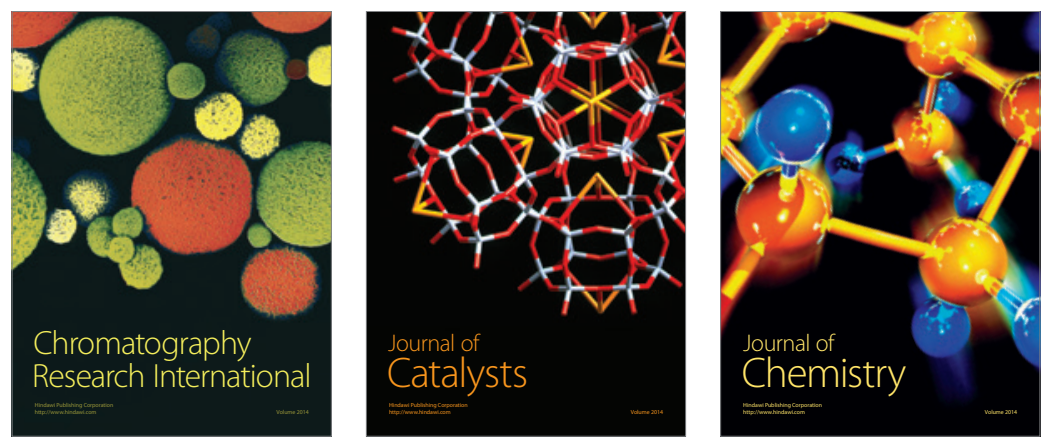
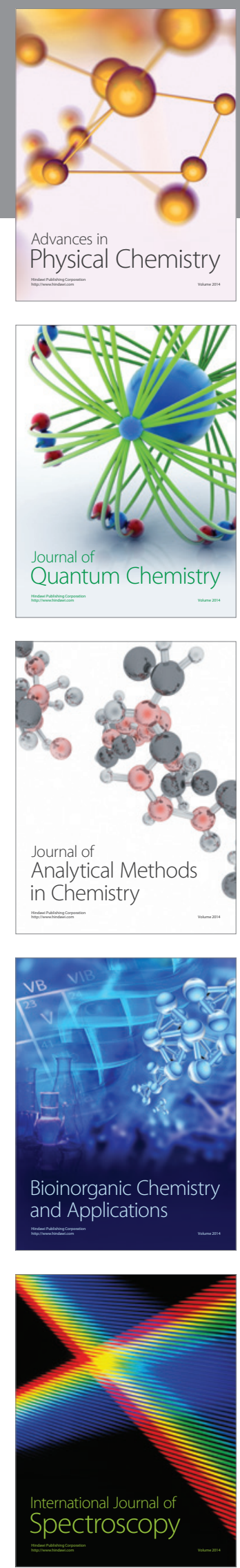